\title{
Introduction to solitons in photonic lattices
}

Nikolaos K. Efremidis ${ }^{1}$, Jason W. Fleischer ${ }^{2}$, Guy Bartal ${ }^{3}$, Oren Cohen ${ }^{3}$, Hrvoje Buljan ${ }^{4}$, Demetrios N. Christodoulides ${ }^{5}$, and Mordechai Segev ${ }^{3}$

1 Department of Applied Mathematics, University of Crete, 71409 Heraclion, Crete, Greece, nefrem@tem.uoc.gr

2 Electrical Engineering Department, Princeton University, Princeton, NJ 08544, jasonf@princeton.edu

3 Physics Department and Solid State Institute, Technion-Israel Institute of Technology, Haifa 32600, Israel, msegev@techunix.technion.ac.il

4 Department of Physics, University of Zagreb, PP 332, Zagreb, Croatia, hbuljan@phy.hr

5 College of Optics and Photonics, University of Central Florida, Orlando, Florida 32813, demetri@creol.ucf .edu

Summary. We present a review on wave propagation in nonlinear photonic lattices: arrays of optical waveguides made of nonlinear media. Such periodic structures provide an excellent environment for the direct experimental observations and theoretical studies of universal phenomena arising from the interplay between nonlinearity and Bloch periodicity. In particular, we review one-dimensional and two-dimensional lattice solitons, spatial gap solitons, and vortex lattice solitons.

\section{Introduction to optical periodic systems}

In this chapter, we present a review of optical systems that have a periodic variation in their index transverse to the direction of propagation. Such photonic systems include photonic crystal fibers, which have a large index variation that controls frequency dispersion, and coupled waveguide arrays, which have a relative small index variation that controls spatial diffraction. Here, we will focus on the latter case and consider $1+1 \mathrm{D}$ and $2+1 \mathrm{D}$ dynamics. A photonic lattice has the advantage that the refractive index contrast requirements are low, and thus, for example, a 2D bandgap can be established for index modulations of the order $10^{-3}$. Light excitation is quite simple because the optical wave is launched in a direction that is almost perpendicular to the direction of the index modulations. Also, nonlinearity can quite easily manifest in such systems simply because of low refractive index modulations. As a result, nonlinear self-localized structures or solitons are possible for nonlinear index modulations of the order of $10^{-4}$.

An optical periodic system with periodicity along the transverse direction was first theoretically studied in 1965 [1] in the linear regime. In that work the diffraction pattern of an array of identical fibers has been found using coupled mode theory in terms of Bessel functions. Experimentally this behavior was reported in 1973 in an array of optical waveguides [2]. 
In 1988, for the first time a nonlinear waveguide array was considered [3]. It was shown that nonlinearity can counteract waveguide coupling, leading to suppressed diffraction and the formation of optical discrete solitons. Discrete solitons were experimentally observed ten years later [4] in AlGaAs arrays.

The transition from one-dimensional to two-dimensional lattices came a few years later. Using an optical induction technique $[5,6]$ allowed for the creation of two-dimensional periodic topologies in photosensitive crystals. This let to the first experimental observation of two-dimensional discrete/lattice solitons in any physical system in nature [7].

During the last years the research interest for studying nonlinear optical periodic systems has grown rapidly (see the recent review articles $[8,9]$ ). There are several factors for this rapid growth in this area. From the physical point of view, the behavior of nonlinearity and periodicity arises in a wide variety of fields, ranging from nonlinear optics and photonic crystals to biology, solid-state physics, and Bose-Einstein condensates in lattice potentials. The common ground in these problems is the co-existence of a band structure along with nonlinearity that in turn allows for inter and intra band interactions.

Considering applications, waveguide lattices are potential candidates for optical switching applications [10, 11, 12, 13]. By engineering regions with different periodicities, which have different band structure properties, it is possible to control of the flow of light. In addition, nonlinearity is a necessary ingredient for performing routing and logic operations.

It has only been recently that the experimental techniques have grown to the point where experiments can be successively performed in two spatial dimensions for a variety of different settings. These methods include, the aforementioned optical induction technique [5, 6] (see section 2 for details), the use of arrays of optical fibers [14], and writing optical waveguides in bulk glasses using femtosecond laser beams [15].

\section{Optically-induced lattices}

Until 2002, waveguide arrays were only fabricated by etching the top of a substrate, creating a series of ridged structures [4]. However, this procedure limits the allowed topologies to only one transverse spatial dimension. Considering applications, higher dimensionality provides the possibility to route information in an optical network from a point of origin A to a final destination Z, something that is not possible using only one spatial dimension [10, 11, 12]. In [5] a new method was suggested to induce a two-dimensional optical lattice in photosensitive crystals. Experimentally the method was first verified in one dimension [6] and subsequently in two-dimensions [7], leading to the first observation of two-dimensional lattice solitons.

Optically induced lattices, are periodic index configurations which are established in biased photosensitive materials. The medium of choice has been 
biased photorefractive crystals, which support both volumetric (holographic) index variations and optical nonlinearity. Let us assume that the crystal is biased with voltage $V$ along the extraordinary crystalline $c$-axis (which is along the $x$ direction). $a$ is the ordinary axis ( $y$ direction) and the optical field propagates along the $z$ direction. Lattice stability requires that the photo-induced crystal is highly anisotropic, being essentially linear and highly nonlinear along the two polarizations. Crystals with such properties are available in photorefractives such as the Strontium Barium Niobate (SBN), which is highly nonlinear along the $c$-axis and essentially linear along the $a$-axis.

Following [16] the normalized propagation of two incoherent beams in a biased photorefractive crystal in one-dimension is given by

$$
\begin{aligned}
& i u_{z}+\frac{1}{2} u_{x x}-\beta_{u}\left[\frac{1}{1+I}-\delta \frac{\partial I / \partial x}{1+I}\right] u=0, \\
& i v_{z}+\frac{1}{2} v_{x x}-\beta_{v}\left[\frac{1}{1+I}-\delta \frac{\partial I / \partial x}{1+I}\right] v=0,
\end{aligned}
$$

ere $u, v$ are the extraordinary and ordinary (respectively) polarized waves, and $\beta_{u} / \beta_{v}=r_{33} / r_{13}$ ( $r_{13}$ and $r_{33}$ are the relevant electro-optic coefficients), $I=|u|^{2}+|v|^{2}$ is the total intensity, and the nonlinearity is of the selffocusing or self-defocusing type if $\beta_{u}, \beta_{v}>0$ or $<0$, respectively. Below, we will establish a simplified model that accurately describes the behavior of the system of Eqs. (1)-(2).

Notice that the term proportional to $\delta$ represents small diffusion effects that can be, in general, ignored. On the other hand, the requirement for large electro-optic anisotropy $r_{33} \gg r_{13}$ results in a parameter region, where the nonlinear term of Eq. 2 can be ignored, leading to the linear diffraction equation for the $v$ field

$$
i v_{z}+\frac{1}{2} v_{x x}=0 .
$$

Thus, propagation along the ordinary axis is essentially linear. Equation (3) admits periodic exact solutions of the form

$$
v=\sum_{j} A_{j} \exp \left(-i \lambda_{j}^{2} z / 2+i \lambda_{j} x+i \phi_{j}\right)
$$

where $A_{j}, \phi_{j}$ and $\lambda_{j}$ are real constants. Experimentally such patterns can be achieved by superimposing plane waves. The only coherent interference pattern in 1D that does not travel in the $x$ direction and remains invariant along $z$ is obtained by interfering two plane waves with $\lambda_{1}=-\lambda_{2}=\pi / L$

$$
v=A \cos (\pi x / L) \exp \left(-i \pi^{2} z /\left(2 L^{2}\right)\right)
$$

Thus, under the above assumptions, Eqs. (1)-(2) describing the evolution of two orthogonally polarized waves can be approximated by 


$$
i u_{z}+\frac{1}{2} u_{x x}-\frac{\beta u}{1+V(x)+|u|^{2}}=0
$$

where

$$
V(x)=A^{2} \cos ^{2}(\pi x / L) .
$$

The field $u$, which is the probe beam, sees a highly nonlinear environment due to the large electro-optic coefficient. Another generic and experimentally feasible model is that of Kerr nonlinear media. The corresponding model equation is given by

$$
i u_{z}+\frac{1}{2} u_{x x}+V(x) u+\sigma|u|^{2} u=0,
$$

where $V(x)$ is proportional to the index modulations and $\sigma= \pm 1$ accounts for self-focusing $(+)$ and self-defocusing ( $(-)$ nonlinearities. Equation (8) describes a variety of optical systems, such as periodic waveguide arrays [4], fiber arrays [14], and Bose-Einstein condensates [17]. In the case of photorefractives, Eq. (8) can be obtained from Eq. (6) in the low intensity limit [assuming $V(x),|u|^{2} \ll 1,-u /\left(1+V(x)+|u|^{2}\right) \approx-u+V(x) u+|u|^{2} u$ ]. Equation (8) is associated with two conserved quantities, namely the total power

$$
P=\int_{-\infty}^{\infty}|u|^{2} d x
$$

and the Hamiltonian

$$
H=\frac{1}{2} \int_{-\infty}^{\infty}\left[\left|u_{x}\right|^{2}-|u|^{4}-2 V(x)|u|^{2}\right] d x .
$$

Thus, Eq. (8) can be written as

$$
i \frac{\partial u}{\partial z}=\frac{\delta H}{\delta u^{*}} .
$$

In two dimensional settings, by employing similar arguments the original model can be simplified to take the form

$$
i u_{z}+\frac{1}{2} \nabla^{2} u-\frac{\beta u}{1+V(x, y)+|u|^{2}}=0,
$$

or for Kerr nonlinear media

$$
i u_{z}+\frac{1}{2} \nabla^{2} u+V(x, y) u+\sigma|u|^{2} u=0 .
$$

In Fig. 1 the experimental configuration of an optical induced lattice is schematically shown. In two spatial dimensions the freedom to select the optically induced lattice is much higher. A generic integral form of the field generated by the interfering beams is given by 



Fig. 1. Configuration of an optically induced lattice. Two pairs of plain waves interfere along the (essentially linear) ordinary polarization, establishing a periodic intensity pattern. On the other hand, the probe beam (red color) polarized along the extraordinary axis propagates in a highly nonlinear environment with a periodic index lattice which is proportional to the intensity pattern of the interfering beams.

$$
v=\iint_{-\infty}^{\infty} A\left(k_{x}, k_{y}\right) e^{i \phi\left(k_{x}, k_{y}\right)} e^{-i\left(k_{x}^{2}+k_{y}^{2}\right) z / 2} e^{i k_{x} x+i k_{y} y} d k_{x} d k_{y}
$$

Notice that the condition for an invariant, along $z$, lattice intensity is satisfied when the "Bloch momentum" vectors $k_{x}, k_{y}$ lie on a circle, i.e., $k_{x}^{2}+k_{y}^{2}=$ $\rho^{2}$. In cylindrical coordinates $k_{x}=\rho \cos \theta, k_{y}=\rho \sin \theta, A\left(k_{x}, k_{y}\right)=\delta(\rho-$ $R) f(\theta) / \rho$, and thus

$$
v=e^{i R^{2} z / 2} \int_{0}^{2 \pi} f(\theta) \exp (i \phi(\theta)) \exp [i R(\cos \theta x+\sin \theta y)] d \theta .
$$

All possible coherent non-diffracting beams can be obtained from Eq.(15) by specifying the amplitude and the phase of the interfering plane waves $[f(\theta), \phi(\theta)]$. Another degree of freedom can be introduced when the lattice is established from mutually incoherent plane waves all of which are polarized along the ordinary axis. In that latter case, the potential is given by

$$
V(x)=\sum_{j}\left|v_{j}\right|^{2} .
$$

where $v_{j} j=1, \ldots, n$ are the mutually incoherent fields, each one of them being determined by an equation of the form (15). Different types of one and two-dimensional diffraction free lattices have been studied in the literature. These include coherent $\left[f(\theta)=\sum_{j=0}^{3} \delta(\theta-j \pi / 2), \phi(\theta)=0\right]$ and incoherent $\left[f_{1}(\theta)=\sum_{j=0}^{1} \delta(\theta-j \pi), f_{2}(\theta)=\sum_{j=0}^{1} \delta(\theta-j \pi+\pi / 2)\right.$, along with $\left.\phi(\theta)=0\right]$ square lattices $[5,7]$, hexagonal lattices $[18,19]$ for which $f(\theta)=\sum_{j=0}^{5} \delta(\theta-$ $j \pi / 3), \phi(\theta)=0$, and Bessel lattices [20,21] with weight functions $f(\theta)=c$ and $\phi(\theta)=m \theta$, where $m$ is the vorticity number. Quasicrystals which are 
structures with long-range order but no periodicity can be formed by the interference of five different plane waves separated by angles $2 \pi / 5$ [22], i.e., $\left[f(\theta)=\sum_{j=0}^{4} \delta(\theta-j 2 \pi / 5)\right.$ and $\left.\phi(\theta)=0\right]$. In Fig. 1(b) the experimental picture of the square lattice intensity pattern established by the coherent interaction of two pairs of orthogonal plane waves is shown.

\section{Coupled-mode theory}

Coupled mode theory (CMT), provides an approximate model for the description of Equations with periodic potentials such as (8) and (13). Although CMT is not exact, it is quite accurate, providing both qualitative and quantitative results, as long as the original assumption from which it was derived are satisfied. Coupled-mode theory originates in the theory of solids, where it is known as tight binding approximation [23]. In optics, it has been extensively used in a variety of linear and nonlinear problems [24, 1, 3]. For simplicity here we consider a one-dimensional model although the same formal analysis can be repeated for higher dimensionalities. CMT is based on the following expansion of the optical field

$$
u=\sum_{n} c_{n}(z) \psi_{n}(x)
$$

In (17) $\psi_{n}(x)=\Psi\left(x-n x_{0}\right)$, where $\Psi(x)$ is the linear lowest order localized mode of a single waveguide in isolation having potential $V_{0}(x)$, i.e.,

$$
q \Psi+\frac{1}{2} \Psi_{x x}+V_{0}(x) \Psi=0 .
$$

Notice that $\Psi$ has zero nodes and is even when the index function is even. In Eq. (17) $c_{n}(z)$ represents the amplitude of the local mode $\psi_{n}$ at distance $z$. Substituting Eq. (17) to (8) an evolution Equation for the amplitudes is derived

$$
i \frac{d c_{n}}{d z}+E c_{n}+\kappa\left(c_{n+1}+c_{n-1}\right)+\gamma\left|c_{n}\right|^{2} c_{n}=0,
$$

where $\kappa=(1 / 2)\left\langle\phi_{n} \mid \phi_{n \pm 1}\right\rangle+\left\langle\phi_{n} \mid V(x) \phi_{n \pm 1}\right\rangle, E=\left\langle\phi_{0} \mid\left(V(x)-V_{0}(x)\right) \phi_{0}\right\rangle$, $\gamma=\sigma\left\langle\phi_{n} \mid \phi_{n}^{3}\right\rangle$, assuming that $\left\langle\phi_{n} \mid \phi_{n}\right\rangle=1$. In a normalized form Eq. (19) reads

$$
i \frac{d c_{n}}{d z}+\left(c_{n+1}+c_{n-1}\right)+\sigma\left|c_{n}\right|^{2} c_{n}=0
$$

where $\sigma$ is the sign of the nonlinearity. Equation (20) is known as the Discrete Nonlinear Schrödinger (DNLS) equation. The regimes of validity of Eq. (20) as an approximate model for periodic systems is discussed in [25]. In particular, DNLS-type models are single band approximations of the lattice NLS equation. Thus, self-defocusing lattice solitons predicted in DNLS models exist in a finite instead of a semi-infinite gap. Notice that coupled-mode theory 
approximations are accurate, as long as the nonlinear index change is much smaller than the linear index modulations. Besides optics, DNLS type models appear in diverse settings such as Biology [26], in molecular crystals [27], in atomic chains [28], and in Bose-Einstein condensates [29] (see also the review papers $[30,31,32])$.

In two transverse dimensions a two-dimensional DNLS Equation is derived

$$
i \frac{d c_{m, n}}{d z}+\left(c_{m, n+1}+c_{m, n-1}+c_{m+1, n}+c_{m-1, n}\right)+\sigma\left|c_{n}\right|^{2} c_{n}=0
$$

for the case of square lattices. For lattices of different symmetries the coupling coefficients can take different form (see for example the case of a honeycomb lattice in [33]).

\section{Linear properties}

A fundamental different between homogeneous and periodic media is that in homogeneous media the dispersion/diffraction curves characterize the regions of continuous/plane wave solutions. On the other hand, media with periodic index modulations do not possess such solutions, but instead support Floquet-Bloch modes. Understanding the linear properties of the system, is not only important per se, but also is fundamental for analyzing the nonlinear properties of the system. In the literature, linear properties have been studied both in the context of the periodic Eqs. (8), (13) or using approximate CMT descriptions (20), (21).

Let us start by considering the approximate, CMT description in onedimension as given by Eq. (20). The discrete Fourier transform of the field amplitude $c_{n}$ is defined by

$$
\tilde{c}_{n}(\kappa)=\frac{1}{\sqrt{2 \pi}} \sum_{n} c_{n}(z) e^{-i \kappa n}, \quad c_{n}(z)=\frac{1}{\sqrt{2 \pi}} \int_{0}^{2 \pi} \tilde{c}_{n}(\kappa) e^{i \kappa n} d \kappa .
$$

The diffraction relation is directly obtained by assuming low amplitude planewave solutions of the form

$$
c_{n}=\exp (-i q z+i k n)
$$

resulting in

$$
q=-2 \cos k
$$

From Eq. (24) the group velocity and the (second order) diffraction are given by $v_{g}=q^{\prime}=d q / d k=2 \sin k$ and $g_{2}=q^{\prime \prime}=2 \cos k$ respectively. Perhaps the most interesting feature of the diffraction relation is the possibility to engineer the second order diffraction term as a function of the incident angle inside the array. For $|k|<\pi / 2$ the diffraction of the array is normal $\left(q^{\prime \prime}>0\right)$, whereas for $\pi / 2<|k|<\pi$ the diffraction becomes anomalous $\left(q^{\prime \prime}<0\right)$. 
Furthermore, for $|k|=\pi / 2$ the second order diffraction term is identical to zero $[34,35]$. Thus a beam propagating with this value of the "Bloch momentum" $k$ is going to experience minimal diffraction, arising only from higher order diffraction terms. The phenomena of negative and zero second order diffraction are not possible in uniform media where the diffraction is always positive (since $q=k^{2} / 2$ ).

The diffraction of optical beams in waveguide arrays when an initial index ramp is applied to the waveguides can be obtained in closed form in the case of discrete models. For the sake of generality, let us consider the linear part of Eq. (20)

$$
i c_{n}+\left(c_{n+1}+c_{n-1}\right)+\gamma n f(z) c_{n}=0
$$

where the linear index ramp across the array $\gamma n$ is multiplied by a function $f(z)$. Equation (25) can be solved in the Fourier domain using the method of characteristics [36]. In particular, assuming single waveguide excitation at the input $\left(c_{m}(0)=\delta_{m, 0}\right)$ the intensity of the optical field along $z$ is given by

$$
I_{m}(z)=\left|c_{m}(z)\right|^{2}=J_{m}^{2}(w)
$$

where

$$
w=\sqrt{u^{2}+v^{2}}, \quad\left(\begin{array}{l}
u \\
v
\end{array}\right)=\int_{0}^{z}\left(\begin{array}{c}
\cos \\
\sin
\end{array}\right)\left[\gamma \eta\left(z^{\prime}\right)\right] d z^{\prime}, \quad \eta=\int_{0}^{z} f\left(z^{\prime}\right) d z^{\prime} .
$$

Because Eq. (25) is linear, the propagation of more complicated patterns is obtained analytically by superposition.

In optics, the diffraction in an array of linear fibers without an additional index tilt $(\gamma=0)$ has been theoretically studied in [1]. In this case, the evolution of a single waveguide excitation is given by $c_{n}(z)=J_{n}(2 z) \exp (i \pi n / 2)$. It is interesting to notice that the amplitude of the field as it propagates is maximum at the edges and not at the center. Experimentally, this behavior has been observed in [2].

A waveguide array with propagation constants that vary linearly in the transverse direction [i.e., $f(z)=1$ in Eq. (25)] exhibits solutions which are called "Bloch oscillations" [37]. Independently of the form of the initial condition, such waves exhibit periodic revivals along $z$, and thus remain localized as they propagate. The required linear variation of the propagation constant is usually achieved by a linear index ramp. Solving Eq. (25) in the special case $f(z)=1$ results to

$$
c_{n}(z)=J_{n}\left(\frac{4}{\gamma} \sin \left(\frac{\gamma z}{2}\right)\right) \exp \left[\frac{i n}{2}(\gamma z+\pi)\right] .
$$

From Eq. (28) one can find that the (intensity) period of the oscillations is equal to $2 \pi / \gamma$. In optics, the existence of Bloch oscillations in waveguide arrays has been predicted in [38]. The experimental observation came one year later independently from two-different groups: In [39] the linear variation of 
the index contract is created by applying a temperature gradient, whereas in [40] the thickness of the central layer is varied. In [34], the diffraction properties of a waveguide arrays were exploited, and it was shown that by using a waveguide array in a zig-zag configuration the second and third order diffraction terms can be canceled out. This can be achieved by using different short segments of waveguides with average zero diffraction. The normal and anomalous refraction and diffraction properties of arrays have been experimentally verified in [35]. AC Bloch oscillations were predicted in [36] $[f(z)$ being a sinusoidal function of $z$ ] in the case where the ratio of the field amplitude and field spatial frequency is a root of the ordinary Bessel function of order 0 . Such periodic modulations in $f(z)$ have not been implemented thus far in optical waveguides. However, it has been shown that an array of optical waveguides whose curvature periodically changes can give rise to AC optical Bloch oscillations [41, 42]. Such periodic oscillations have been observed in $[43,44]$.

Coupled mode theory equations are single band approximations of Eqs. (8), (13) which exhibit a periodic potential. To consider higher-band behavior, these equations can be analyzed by making use of Floquet-Bloch theory. Let us consider the linear two-dimensional case. Looking for wave solutions, whose amplitude is stationary along $z$, i.e., $u(x, y, z)=\psi(x, y) \exp (-i q z)$, we obtain

$$
q \psi_{z}+\frac{1}{2}\left(\psi_{x x}+\psi_{y y}\right)+V(x) \psi=0 .
$$

Floquet-Bloch's theorem [45, 37, 23] states that the eigenfunctions of Eq.(29) for a periodic potential are the products of a plane wave $\exp (i \mathbf{k} \cdot \mathbf{r})$ multiplied with a function $\Psi_{\mathbf{k}}(\mathbf{r})$ with the periodicity of the crystal lattice, or

$$
\psi_{\mathbf{k}}=\Psi_{\mathbf{k}}(\mathbf{r}) e^{i \mathbf{k} \cdot \mathbf{r}}
$$

where $\Psi_{\mathbf{k}}(\mathbf{r}+\mathbf{R})=\Psi_{\mathbf{k}}(\mathbf{r}), \mathbf{R}=m \mathbf{R}_{1}+n \mathbf{R}_{2}, m, n \in \mathbb{Z}$ and $\mathbf{R}_{1}, \mathbf{R}_{2}$ are the primitive vectors of the lattice such that $V(\mathbf{r}+\mathbf{R})=V(\mathbf{r})$. A related concept is that of a Brillouin zone which is defined as the primitive cell in the reciprocal lattice. Several methods have been developed in condensed matter physics [23] to find the band structure of Eq. (29). A simple approach, known as the plane-wave method, relies on the Fourier series decomposition of $\Psi_{\mathbf{q}}(\mathbf{r})$ under periodic boundary conditions. The resulting system of algebraic equations can then be solved numerically. Some specific types of potentials admit exact solutions, such as the Kronig-Penney model in one-dimension [46] and the sinusoidal potential which can be solved in one or higher dimensions with the use of Matheiu functions [47].

Let us consider the one-dimensional case with a typical sinusoidal potential of the form

$$
V(x)=-V_{0} \sin ^{2}(\pi x / 2) .
$$

The period of the potential is 2 and its band structure is shown in Fig. 2 for $V_{0}=10$ in the reduced zone scheme (the band structure is "folded" 


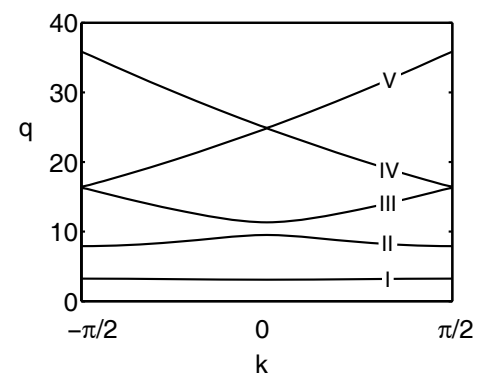

Fig. 2. A typical one-dimensional band structure for the lattice potential of Eq. (31) for $V_{0}=10$. Curves I-V correspond to the first five bands of the complete band structure.

inside the first Brillouin zone). The bands of Fig. 2 are the regions where periodic Floquet-Bloch modes exist, i.e. modes that have allowed values of the propagation constant $\mathrm{q}$. The regions outside the bands are called bandgaps. Inside the bandgaps exponentially decaying/growing modes are supported. These modes can be obtained for example using the plane-wave method for complex values of the Bloch momentum. Notice that between two successive bands, a bandgap always exist in the $q$ domain. In waveguide arrays, the band structure and the corresponding Floquet-Bloch modes were investigated in [48] for one spatial dimension.
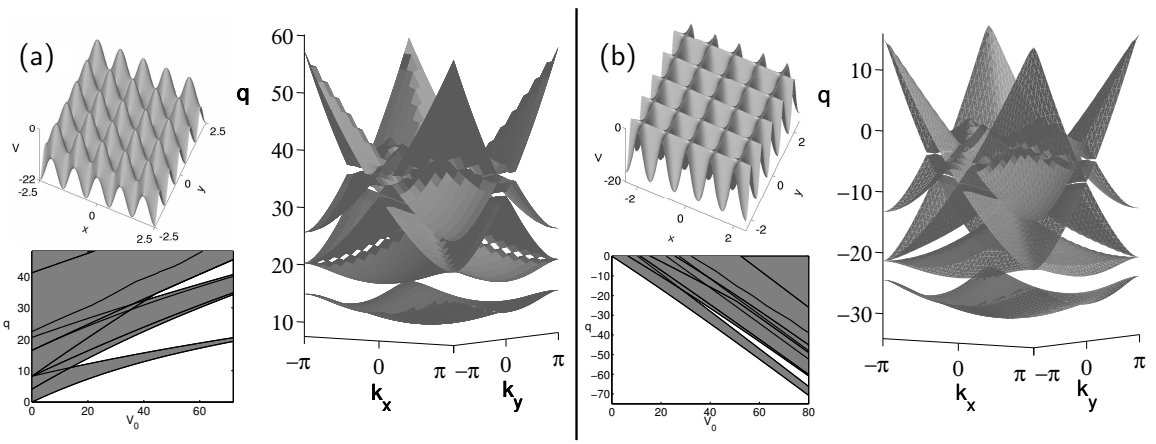

Fig. 3. Index potentials of Eqs. (32) and (33) and their correspond band structure properties are shown in (a), (b), respectively. The numerically calculated band structures for (a) $V_{0}=21.6$ and (b) $A^{2}=1.21$ and $V_{0}=-36.3$ are shown. The regions of bands (gray) and bandgaps (white) are depicted as a function of the potential depth.

The band structure properties become more complicated in more than one dimension. We analyze two different type of lattices [49]. The first index potential is sinusoidal [Fig. 3(a)] 


$$
V(x, y)=-\left(V_{0} / 2\right)\left[\sin ^{2} \pi x+\sin ^{2} \pi y\right],
$$

whereas, the second lattice has a backbone index profile

$$
V(x, y)=-\frac{V_{0}}{1+A^{2} \cos ^{2}(\pi x) \cos ^{2}(\pi y)} .
$$

The lattice of Eq. (33) [shown in Fig. 3(b)], is established by the interference of two pairs of plane waves that are coherently superimposed.

A complete bandgap is defined as a finite region in $q$ between two successive (in increasing $q$ order) Floquet-Bloch mode. As can be seen from the three-dimensional band structure shown in Fig. 3, different bands can overlap with each other in the $q$ axis restricting, or even eliminating altogether, the number of complete bandgaps. This property of higher dimensional lattices is in contract to the one-dimensional arrays, which are, in general, associated with an infinite number of complete Bragg resonance bandgaps. In Fig. 3 we display the band (gray) and bandgap (white) regions for varying potential depth. In Fig. 3(a) we find that no complete bandgap exist for $V_{0} \lesssim 13.8$. By increasing the potential depth further, a second bandgap opens up when $V_{0} \simeq 40.7$. The backbone lattice of Fig 3(b) exhibits different behavior. When the potential depth is less than 28.4 (and $A^{2}=1.21$ ) all the bands overlap, i.e. no complete band gap exists. On the other hand, for bigger values of $V_{0}$, one gap opens up between the first and the second bands. Unlike the sinusoidal lattice case, we find that no other band gaps emerge for even greater values of $V_{0}$.

The presence of a linear index ramp (say $\gamma x+\delta y$ in two spatial dimensions) in addition to the periodic lattice is expected to give rise to Bloch oscillations. However, Zener predicted [50] that Bloch oscillations are not ideal (they persist for finite distances) in periodic lattices due to interband interactions. Furthermore, Bloch oscillations are expected to breakdown when then index difference imposed on a period of a lattice due to the linear potential is of the order of the gap to the next band. Zener tunneling in one-dimensional waveguide arrays has been observed in [51]. In [52] Bloch oscillations and Zener tunneling in two-dimensional periodic systems have been reported. In this latter work, an optical induction technique was applied and, in order to create a transverse refractive index gradient, the crystal was illuminated from the top with incoherent white light.

A method of optical waveguiding, which relies on Bragg diffractions from a 1D grating that gives rise to waveguiding in the direction normal to the grating wave vector was proposed in [53]. The waveguide structure consists of a shallow 1D grating that has a bell- or trough-shaped amplitude in the confinement direction. In [54] nondiffracting beams in two-dimensional periodic systems were identified. Such beams are constructed by superposition of Floquet-Bloch modes.

In dispersion curves, there are specific points known as diabolic points which are singular. In [18] it was demonstrated that such a diabolical point 
exists in honeycomb lattices, around which an input beam experiences conical diffraction.

\section{One-dimensional lattice solitons}

In the early works on optical discrete solitons, the theoretical analysis was based on CMT description. This single band model, can provide valuable information about the behavior of optical periodic systems (8), (13). In onedimension Eq. (20) is not integrable unlike its continuous analogue, the nonlinear Schödinger equation. An integrable version of the DNLS equation, which is known as the Ablowitz-Ladik DNLS exists [55].

We are going to analyze basic families of discrete soliton solutions by utilizing the DNLS equation in one transverse dimension. We assume that Eq. (20) admits solutions of the form

$$
c_{n}=a_{n} \exp (-i q z),
$$

and thus

$$
q a_{n}+\left(a_{n+1}+a_{n-1}\right)+\sigma a_{n}^{3}=0 .
$$

Asymptotic analysis of soliton solutions can be carried out in the limit of nonlinear modes which are highly localized inside the lattice. We are going to analyze two basic families of discrete solitons of Eq. (34) known as "on-site" and "off-site". We isolate these solutions from many other types because, by analytic continuation, they represent single hump soliton solutions in the long wavelength limit. In the strongly nonlinear limit the first family of solutions [3] has the approximate form

$$
a_{0}=\alpha, \quad a_{ \pm 1}=1 /(\sigma \alpha),
$$

whereas $u_{n}=0$ for $n \neq 0, \pm 1$ and $q=-\sigma \alpha^{2}$. This family of solution is called "on-site" because the maximum of an imaginary envelope is located exactly at the lattice site $n=0$ [Fig. 4]. The second family of solutions is given by

$$
\begin{gathered}
a_{j}=\alpha(\operatorname{sgn}(\sigma))^{j}, \quad j=0,1, \\
a_{j}=\alpha(\operatorname{sgn}(\sigma))^{j} /\left(1+|\sigma| \alpha^{2}\right), \quad j=-1,2,
\end{gathered}
$$

and $q=-\operatorname{sgn}(\sigma)\left(1+|\sigma| \alpha^{2}\right)$. This family of solutions is called "intra-site" or "off-site" because an imaginary envelope has its maximum between lattice sites 0 and 1 [56]. For both families of solitons $\operatorname{sgn}(\sigma)=-\operatorname{sgn}(k)$. The "onsite" family of solitons is energetically favorable, and thus stable whereas "intra-site" discrete solitons are unstable [57, 58]. The exponential decay (for large $|n|$ ) of these solutions is given by

$$
a_{n}=(\operatorname{sgn}(\sigma))^{n} \exp (-\lambda n) .
$$




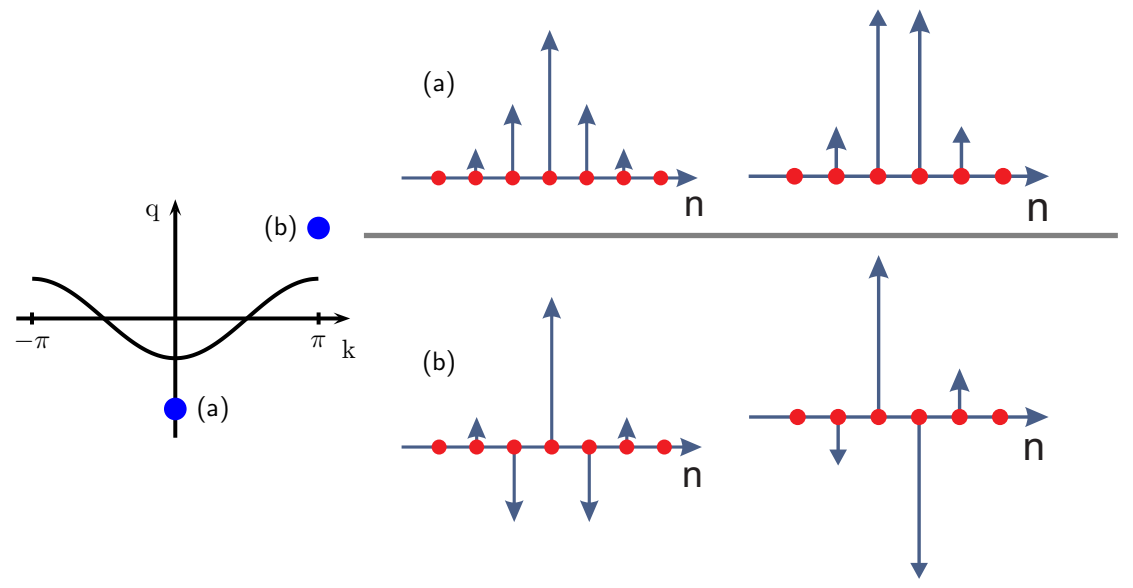

Fig. 4. Diffraction relation (left) and families of discrete solitons supported by the DNLS equation. The two families shown in (a) ["on-site" (left) and "off-site" (right)] exist for self-focusing nonlinearities and have eigenvalues in the bandgap below the base of the 1st Brillouin zone. The families shown in (b) ["on-site" (left) and "off-site" (right)] exist for self-defocusing nonlinearities and have eigenvalues in the bandgap above the edge of the 1st Brillouin zone.

where $\lambda=\operatorname{arccosh}(k / 2)$.

Notice that these two families of solutions exist for both signs of the nonlinearity [59]. This result is in contrast to the nonlinear Schrödinger Equation which admits bright soliton solutions in the self-focusing case only. In Fig. 4 these two families of discrete solitons are schematically illustrated for selffocusing [Fig. 4(a)] and self-defocusing [Fig. 4(b)] nonlinearity. We would like to point out that for self-focusing nonlinearity $(\sigma>0)$ the soliton eigenvalue resides in the semi-infinite bandgap below the 1st Brillouin zone $(q<-1)$. On the other hand, self-defocusing solitons $(\sigma<0)$ have eigenvalues residing above the Brillouin zone $(q>1)$. In addition, the phase difference between adjacent lattice sites of self-focusing discrete solitons is zero, and thus these solitons reside at the base of the Brillouin zone $(k=0)$; The adjacent lattice site phase difference of self-focusing discrete solitons is $\pi$, i.e., these solitons reside at the edge of the Brillouin zone $(k=\pi)$. The mathematical proof for the existence of discrete solitons was derived in [60].

The analysis of lattice solitons in periodic lattices beyond the DNLS limit, reveals that the behavior of the system is more complex [5, 61, 25]. Families of lattice solitons (LS) can exist in the semi-infinite [or total internal reflection (TIR)] gap and in the finite (Bragg) bandgaps of the band structure. Thus, an infinity of families of LS in principle exist in one-dimensional periodic lattices. In the case of self-focusing nonlinearity [Fig. 5(a)] families of lattice solitons exist both in the TIR gap and in every Bragg resonance gap. A 
(a)
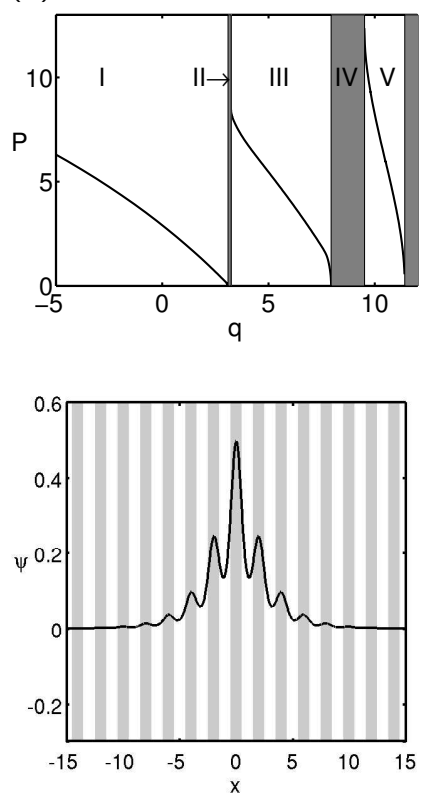

(b)
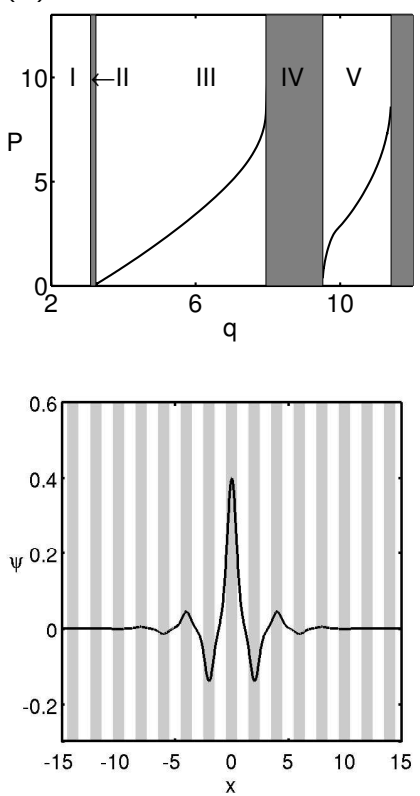

Fig. 5. Lattice solitons existence curves (total power $P$ vs. propagation constant $q$ ) for self-focusing (a) and self-defocusing (b) Kerr media. The gray (shaded) regions II and IV depict the first two bands. Regions I, III, IV correspond to the semiinfinite and the first two Bragg (finite) gaps of the system. Typical soliton profiles with an eigenvalue in the semi-infinite gap (a) and in the first finite bandgap (b) are shown on the bottom. Gray (white) areas represent high (low) refractive index regions.

typical TIR lattice soliton is shown on the bottom of Fig. 5(a). Notice the absence of nodes (the field does not become zero) in the profile which is characteristic for TIR LS. In Fig. 5(b) the existence curves of self-defocusing lattice solitons are shown [25]. In this case lattice solitons do not exist in the TIR bandgap, but only in the Bragg gaps. The field profile of a typical lattice soliton with eigenvalue inside the first Bragg resonance is shown on the bottom of Fig 5(b).

Figure 6 depicts experimental results showing lattice soliton formation in optically induced lattices [6]. In Fig. 6(I) the transition of the signal beam from discrete diffraction to a discrete soliton for on-axis (corresponding to zero Bloch momentum, or at the base of the 1st Brillouin zone) input as a function of self-focusing nonlinearity is depicted. When the nonlinearity is small, the signal beam experiences diffraction. On the other hand, in the strongly nonlinear regime, a highly localized lattice soliton is formed [Fig. 6(I)(e)-(f)]. This family of solutions reside in the semi-infinite bandgap 
(I)

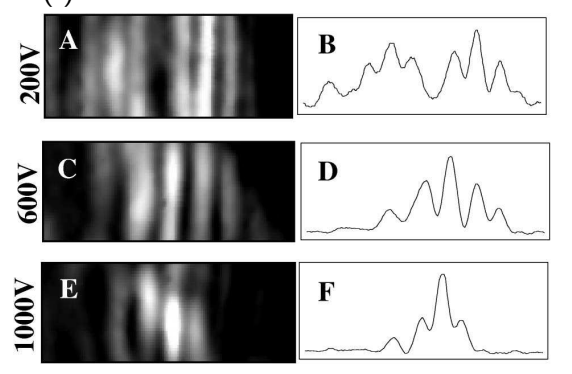

(II)

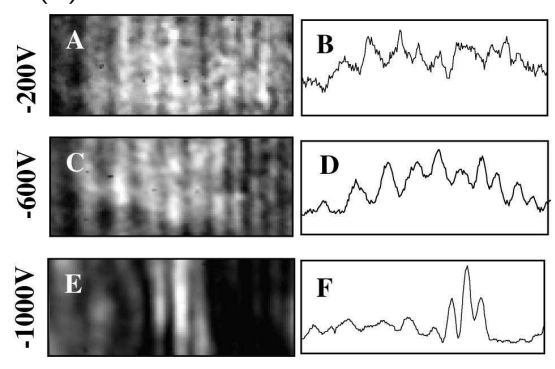

Fig. 6. Signal beam output intensity as a function of increasing focusing (I) and defocusing (II) nonlinearity. (A)(B) show discrete diffraction (C)(D) show intermediate self-focusing and $(\mathrm{E})(\mathrm{F})$ depict lattice soliton formation. The period of the waveguide lattice is $8.8 \mu \mathrm{m}$ and $9.3 \mu \mathrm{m}$ in (I) and (II), respectively.

of the band structure. Such solitons were first observed in optical waveguide arrays in [4]. Figure 6(II) shows lattice soliton formation when the signal beam is incident at an angle very close to the first Bragg resonance (or at the edge of the 1st Brillouin zone) of the system with self-defocusing nonlinearity. When the nonlinearity is small, the beam diffracts. Increasing the applied voltage (thus increasing the nonlinearity) a self-defocusing gap soliton residing in the first bandgap of the band structure is formed. This family of solitons was first observed in [6].

There is a plethora of works based on DNLS type Equations and, during the last years, on lattice NLS equations. Pairs of out-of-phase solitons, which resemble twisted localized modes were observed in [62]. Self-focusing gap solitons were reported in waveguide arrays in [63]. Properties of gap solitons such as Bloch wave interactions [64] and controlled generation and steering [65] have also been studied.

Modulational instability of plane wave solutions has been theoretically analyzed in $[3,66]$. Modulational instability was observed in AlGaAs waveguide arrays with self-focusing nonlinearity [67]. Such instabilities occur as long as the spatial Bloch momentum of the initial excitation is within the normal diffraction region of the Brillouin zone. Modulation instability in the anomalous-diffraction regions of a photonic lattice has been observed in [68]. The experiments were carried out in a $1 \mathrm{D}$ waveguide array fabricated in a lithium niobate crystal displaying the photovoltaic self-defocusing nonlinearity.

Another basic family of solutions supported by a lattice is that of dark solitons. Dark discrete solitons have the form of a dip in a uniform background of light. Theoretically, their properties have been studied in the discrete domain [69]. Experimentally, they have been observed in self-focusing waveguide arrays, that support dark gap solitons $[70,63]$. Notice that in order to excite a 
dark soliton, a $\pi$ step needs to be introduced at the center of the interference pattern that results in a dark narrow notch in the shaped input beam.

The propagation of two different waves, which can be optical fields of different colors or polarizations or mutually incoherent beams, is described by a system of coupled nonlinear Schrödinger equations. Solutions of such models are known as vector solitons [71]. The families of vector solitons in discrete lattices have been analyzed and their stability has been studied in [72] for strongly localized modes. Experimentally, discrete vector solitons were reported in [73] in Kerr nonlinear waveguide arrays. The vector elements consisted of two coherently coupled orthogonal polarizations. In spite of fourwave mixing effects, such solitons were found to be stable. In the continuous periodic model [74] or in discrete superlattices [75] the presence of multiple Bragg gap can allow for more complicated localized vector structures, such as multigap vector solitons, which have components with eigenvalues in different gaps of the band structure.

Another two-component vector family is that of quadratic solitons. In such media, the fundamental and the second harmonic interact to form a localized soliton solution. Theoretically, discrete quadratic solitons were analyzed in [76]. Discrete solitons with two frequency components mutually locked by a quadratic nonlinearity have been observed in [77]. Experiments have been performed in waveguide arrays with tunable quadratic nonlinearity.

Spatiotemporal effects have been analyzed theoretically in [78] and experimentally in [79]. Temporal dispersion results to a sharp transition from strong diffraction at low powers to strong localization at high powers. In [80] it was shown that two components consisting of a periodic and a localized wave, such as that of an optical lattice, are exact vector soliton solutions of the system. The analytic form of the solution was derived in [81]. Families of exact solutions were also derived in [82] for the case of a linear-nonlinear structure.

Recently, the study of discrete/lattice solitons at interfaces has attracted considerable attention. Such solutions were first predicted to exist at the edge of an array above a certain power threshold [83]. Surface lattice solitons can also exist in the Bragg gap of an optical lattice [84]. Experimentally, surface solitons were observed in [85] at the interface between a nonlinear self-focusing waveguide lattice and a continuous medium. Surface gap solitons were observed in [86].

Discrete solitons traveling in the transverse plane are known to decelerate in periodic systems due to the presence of the Peierls-Nabaro potential [56, 87]. In [88] it was shown that by using a special prechirped ansatz traveling waves are more robust as compared to regular linear chirp. In [89] it was shown that in discrete systems with saturable nonlinearity traveling modes can exist for specific values of the spectrum. Such solutions can be considered as embedded solitons. 
Two-dimensional $\mathrm{X}$-wave nondiffracting solutions are known to exist in linear bidispersive optical systems [90]. This family of optical waves has been excited in waveguide arrays, by using the interplay between discrete diffraction and normal temporal dispersion, in the presence of Kerr nonlinearity [91].

In the case of a periodic potential with a low-index defect, localized defect modes exist as a result of repeated Bragg reflections [92]. Strongly confined defect modes appear when the lattice intensity at the defect site is nonzero rather than zero. Furthermore, it is possible to construct a waveguide lattice that relies on the effect of bandgap (Bragg) guidance, rather than total internal reflection, in the regions between defects [93]. In the nonlinear regime the Kerr effect can counteract diffraction leading to the formation of gap lattice solitons.

Another family of solutions is that of dissipative discrete solitons. Such solitons were studied first in the context of the discrete cubic-quintic GinzburgLandau equation [94]. In that work, the basic families of solutions and their stability were analyzed. Dissipative lattice solitons were also studied theoretically in waveguide array configurations that involve periodically patterned semiconductor optical amplifiers and saturable absorbers [95]. Exact solutions for dissipative discrete solitons can be found, when the discretization of the Ginzburg-Landau equation is similar to the Ablowitz-Ladik model [96].

The theoretical and experimental investigation of optical beam interactions was reported on [97]. Discrete solitons in periodic diffraction managed systems were studied in [98]. Lattice solitons have been studied in other settings, such as nematic liquid crystals [99] which are supported due to a nonlocal nonlinearity. For reviews on the properties of one-dimensional discrete solitons see also $[8,100,101,102]$.

\section{Two-dimensional lattice solitons}

Two-dimensional settings allow better control of the flow of light as compared to a planar geometry. For example, in $[10,11,12]$ it was shown theoretically that discrete solitons can be navigated in two-dimensional networks of nonlinear waveguide arrays. This can be accomplished via vector interactions between two classes of discrete solitons: signals and blockers. Discrete solitons in such two-dimensional networks can exhibit a rich variety of functional operations, e.g., blocking, routing, logic functions, and time gating.

Following [49], we are going to analyze basic properties of solitons in 2D periodic lattices. In Fig. 7 typical existence curves as well as field profiles of two-dimensional LS are shown. The self-focusing soliton shown in Fig. 7(a) exists in the semi-infinite (TIR) bandgap below the first band. On the other hand, the self-defocusing solitons shown in Fig. 7(b) exist in the finite (Bragg) bandgap between the first and the second band. It is important to notice that a complete bandgap is always required for gap lattice solitons to exist; i.e., shallow potentials do not support gap lattice solitons. If the bandgap is only 
(a)
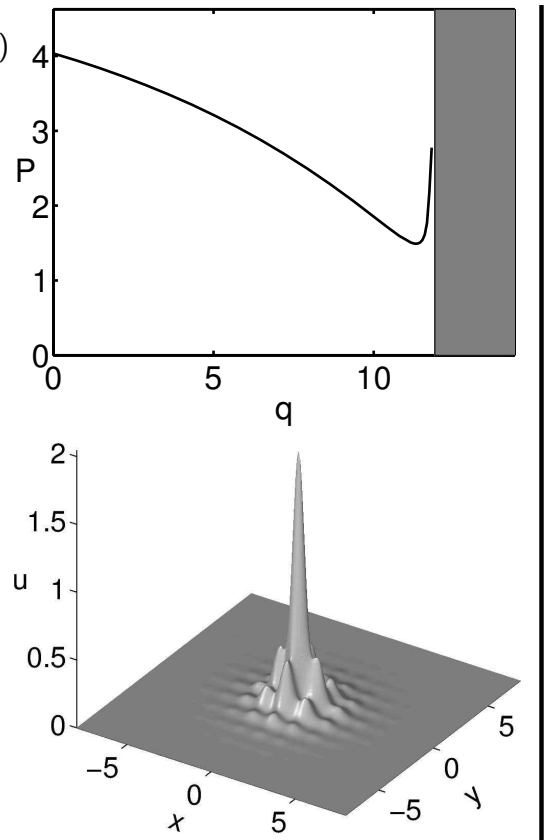

(b)
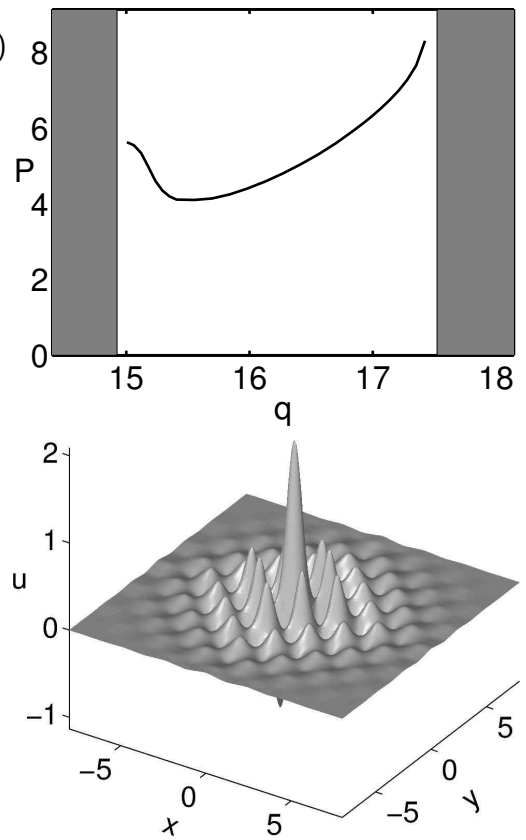

Fig. 7. The existence (power vs. eigenvalue) curves (top) for self-focusing (a) and self-defocusing (b) Kerr nonlinearity. Gray areas represent bands. On the bottom typical field profiles of these soliton families are depicted.

partial (a situation not encountered in 1D), an input beam will radiate due to the interactions with the linear spectrum.

The power $P$ conveyed by the solitons versus the eigenvalue $q$ is shown on the top of Fig. 7. Note that there is a minimum power threshold required in order to observe a lattice soliton (in a finite or infinite bandgap) in two dimensions. In the $1 \mathrm{D}$ case, such a threshold does not exist [25, 61] (see Fig. 5). In the case of a semi-infinite band gap these results are in agreement with the discrete nonlinear Schrödinger case $[103,104]$ as rigorously proven in [105].

The existence curve also provides information on the stability of the solitons. For the self-focusing case the stability can be determined by a straightforward application of the Vakhitov-Kolokolov criterion [106]. More specifically, when $\partial P / \partial q<0$ the solutions are stable, while, close to the band $\partial P / \partial q>0$ and the lattice solitons become unstable. This analysis cannot be applied directly to the defocusing case (the soliton amplitude has nodes).

In a Kerr nonlinear NLS equation in two (critical) or three (supercritical) spatial dimensions an input beam collapses in finite time to a singularity. A characteristic property of DNLS lattices is the absence of collapse irrespec- 
tively of the dimensionality of the problem [107]. However, this behavior does not always convey to periodic systems [108]. Specifically, when the soliton is highly confined into the lattice, it becomes unstable in the supercritical case. In the critical case, although the soliton is mathematically stable, its basin of attraction is so small that the lattice soliton is physically unstable. On the other hand, broad solitons in critical and supercritical dimensions can be stable if the lattice confinement is high, in agreement with the discrete model.

(a)


(b)

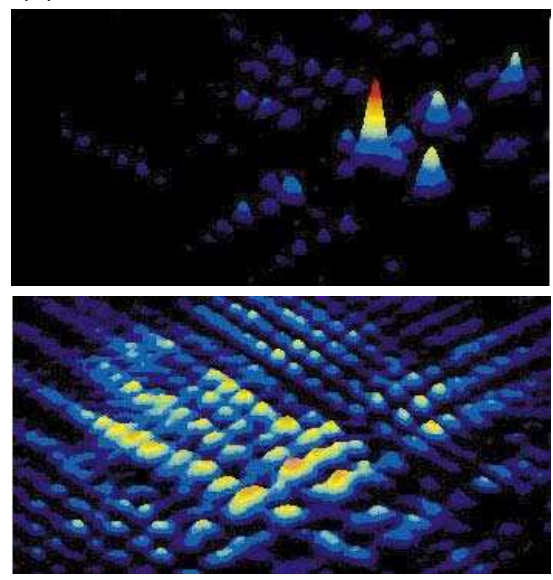

Fig. 8. Experimental results depicting the intensity structure of the probe beam at the exit facet of the $6 \mathrm{~mm}$ long crystal. (a) self-focusing nonlinearity; discrete diffraction at low intensity (bottom), and formation of self-focusing TIR lattice solitons at high intensities (top). (b) self-defocusing nonlinearity; discrete diffraction at low intensity (bottom), formation of self-defocusing gap lattice solitons at high intensities (top).

Following [7] experimental results of $2 \mathrm{D}$ lattice solitons are shown in Fig. 8. The waveguide arrays is induced in real time, in a photosensitive material from two pairs of plane waves. A separate "probe" beam is launched into the periodic waveguide array. The experimental results for self-focusing nonlinearity are shown in Fig. 8(a). The "Bloch momentum" of the probe beam is zero (base of the 1st Brillouin zone). The probe beam is launched into a single waveguide. At low voltages, the beam propagates, essentially, linearly and as a result discrete diffraction concentrates the signal intensity into the outer perimeter of a square [Fig. 8(a) bottom]. For a stronger nonlinearity (at higher voltage), self-focusing dominates and a lattice soliton is formed [Fig. 8(a) top]. An interferogram of this soliton (not shown), obtained by interfering the soliton output beam with a plane wave, shows constructive 
interference of all the elements; that is, the central peak is in-phase with its neighbours.

The formation of self-defocusing lattice solitons is shown in Fig. 8(b). In this case the Bloch momentum of the "probe" beam lies in the vicinity of the M point of the first Brillouin zone, so as to excite gap lattice solitons with eigenvalues inside the first bandgap. At low voltages, a diffuse diffraction pattern occurs [Fig. 8(a) bottom], while a self-defocusing gap lattice soliton is observed [Fig. 8(a) top] at a higher nonlinearity. Self-focusing solitons in the TIR regime have also been shown in [109]. An interferogram (not shown) confirms the $\pi$ phase difference between first neighbors: the central peak is lowered while the surrounding lobes increase their intensity, indicating destructive and constructive interference, respectively.

Interaction of a 2D lattice soliton with a lattice was shown in [110]. In this work, other phenomena such as lattice dislocation, and creation of structures akin to optical polarons were demonstrated. Two-dimensional gap lattice solitons can also exist in the presence of self-focusing nolninearity. Such a family of solutions was studied theoretically and experimentally in [111].

Two-dimensional lattice solitons at interfaces have been studied theoretically in $[112,113]$. Experimentally, surface lattice solitons were observed at the boundaries of a finite optically induced photonic lattice [114] and at the edge of femtosecond laser-written waveguide arrays in fused silica [115].

Families of two-dimensional dissipative Ginzburg-Landau solitons have been studied in [116]. Discrete solitons and their stability in Honeycomb lattices were examined theoretically in [33]. Two-dimensional TIR and gap solitons in such lattices were observed in $[18,19]$. In square lattices, dipole like modes [117] and two-dimensional lattice vector solitons [118] have been studied theoretically and experimentally.

Nondiffractive rotary Bessel lattices can support families of localized waves. In particular, in addition to the lowest order soliton trapped in the center of the lattice, solitons can be trapped at different lattice rings [20, 21].

Quasicrystals are structures with long range order but no periodicity. The lack of periodicity excludes the possibility of describing quasicrystals with analytical tools, such as Bloch's theorem and Brillouin zones. In [22] it was demonstrated that light launched at different quasicrystal sites travels through the lattice in a way equivalent to quantum tunneling of electrons in a quasiperiodic potential. At high intensities lattice solitons are formed.

Anderson localization theory predicts that an electron may become immobile when placed in a disordered lattice. The origin of localization is interference between multiple scatterings of the electron by random defects in the potential altering the einenmodes from being extended (Floquet-Bloch waves) to exponentially localized. In [119] the experimental observation of Anderson localization in a perturbed periodic potential was reported. 


\section{Vortex solitons in lattices}

Vortex solitons are self-localized solutions of nonlinear wave equations, which are characterized by a phase singularity at the pivotal point. The phase charge of a simple closed curve surrounding the vortex core is equal to $2 \pi m$, where $m$ is the integer vorticity of the solution.

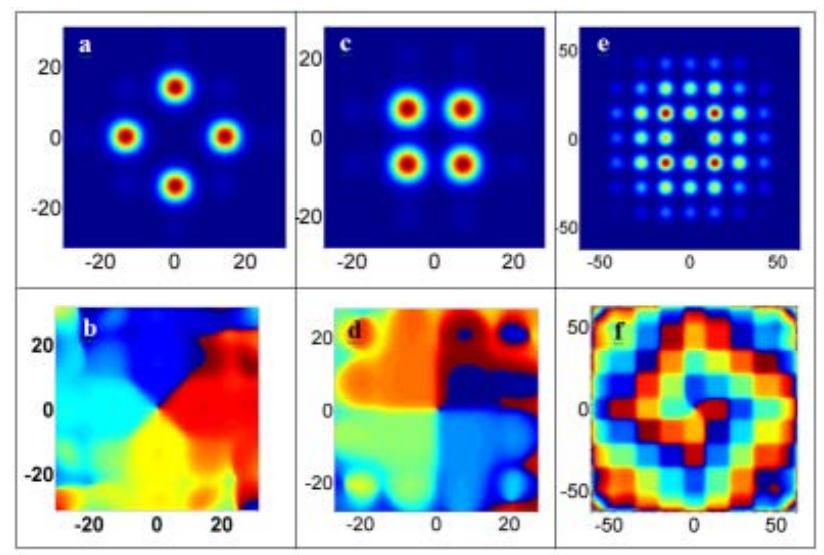

Fig. 9. Calculated intensity and phase of (a),(b) the on-site and (c),(d) the offsite vortex lattice solitons, along with the output diffraction pattern of (e), (f) the off-site vortex at $z=800$.

The optical case of discrete vortices was considered in Kerr nonlinear waveguide arrays, where on-site vortices (vortices whose singularity is located on a lattice site) [120] and off-site vortices (vortices whose singularity is located between sites) $[109,121]$ were studied. Both cases were found to be stable within a certain range of parameters. Experimental results on optical vortex solitons were presented in [122, 123]. Here, we present results from [122].

Typical beam propagation results showing on-site and off-site vortex solitons for $z=800$ are given in Figs. 9(a)-9(d). The main four "lobes" all have the same peak intensity and, importantly, each lobe is $\pi / 2$ out of phase with its neighbors. Note again that the singularity of the on-site vortex is centered on a lattice site [Figs. $9(\mathrm{a})$ and $9(\mathrm{~b})$ ], whereas the singularity of the off-axis vortex is centered between four lattice sites [Figs. 9(c) and 9(d)]. The soliton exhibits stationary propagation, and the shapes of the vortices remain unchanged; i.e., these are, indeed, vortex lattice solitons. For comparison, when the nonlinearity is set to zero, the vortices diffract by tunneling between lattice sites, as shown in Figs. 9(e) and 9(f) for the off-site vortex after $z=800$. Note that the phase of the diffracting beam maintains its spiral structure throughout diffraction [Fig. 9(f)]. 




Fig. 10. Experimental results at the output face of crystal. (a),(b) Diffraction at $100 \mathrm{~V}$ : (a) intensity and (b) phase information formed by interference of output with a plane wave. (c) Typical on-site soliton at $700 \mathrm{~V}$, and (d) relative phase from an interferogram. (e) Typical off-site soliton at $700 \mathrm{~V}$, and (f) relative phase from an interferogram. Note that phase information is extremely sensitive to background noise, so only the interference patterns from the relevant lattice sites are shown.

Experimental results are shown in Fig. 10. The photorefractive screening nonlinearity is controlled by applying voltage against the crystalline $\mathrm{c}$ axis. At a low voltage $(\sim 100 \mathrm{~V})$, the output diffraction of both the on-site and off-site vortices looks similar. Figures 10(a) and 10(b) show the diffraction pattern of an on-site vortex after $5 \mathrm{~mm}$ of propagation, showing that both the hole and the width of the ring expand through the lattice. The ring expands to roughly 3 times its original size [Fig. 10(a)], while an interferogram, created by interfering the output pattern with a plane wave, clearly shows the $0 \rightarrow 2 \pi$ spiral phase structure of the vortex [Fig. 10(b)]. At around $700 \mathrm{~V}$, the input vortices become vortex lattice solitons and maintain their structure (intensity and phase) while propagating. Figures $10(\mathrm{c})$ and $10(\mathrm{~d})$ show the intensity and phase of an on-site soliton, while Figs. 10(e) and 10(f) show those features for an off-site soliton. Because phase information is very sensitive to noise in the system, only the relative phase of the relevant (soliton) lattice sites is shown for clarity.

Other families of vortices have been subsequently studied. These include higher-band vortex solitons [124, 125], asymmetric vortex solitons [126], and multivortex solitons in triangular photonic lattices [127].

\section{Random-phase lattice solitons}

This chapter has dealt with coherent nonlinear phenomena in discrete lattices. It should be pointed out that many interesting nonlinear phenomena 
with partially incoherent light in photonic lattices have been discovered in the past few years. Lattice solitons made of partially-incoherent light were theoretically predicted in [128]. The first experimental observation of such solitons [129] has shown that due to the simultaneous excitation of multiple bands, and the interplay of dispersion in a lattice and the nonlinearity, the spatial power spectra of the observed solitons is multi-humped, with humps

being located in the normal diffraction regions for self-focusing nonlinearity and vice versa for the self-defocusing nonlinearity. The dynamics of incoherent light in nonlinear lattices, which leads to such intricate structures has lead to a technique for Brillouin zone spectroscopy [130], and studies of other nonlinear phenomena with incoherent light in photonic lattices (e.g., see [131] and Refs. therein).

\section{References}

1. A. L. Jones. "Coupling of optical fibers and scattering in fibers." J. Opt. Soc. Am. 55, 261-271 (1965).

2. S. Somekh, E. Garmire, A. Yariv, H. Garvin, and H. R.G. "Channel optical waveguide directional couplers." Appl. Phys. Lett. 22, 46-47 (1973).

3. D. N. Christodoulides and R. I. Joseph. "Discrete self-focusing in nonlinear arrays of coupled waveguides." Opt. Lett. 13, 794-796 (1988).

4. H. S. Eisenberg, Y. Silberberg, R. Morandotti, A. R. Boyd, and J. S. Aitchison. "Discrete spatial optical solitons in waveguide arrays." Phys. Rev. Lett. 81, 3383-3386 (1998).

5. N. K. Efremidis, D. N. Christodoulides, S. Sears, J. W. Fleischer, and M. Segev. "Discrete solitons in photorefractive optically-induced photonic lattices." Phys. Rev. E 66, 046602 (5 pages) (2002).

6. J. W. Fleischer, T. Carmon, M. Segev, N. K. Efremidis, and D. N. Christodoulides. "Observation of discrete solitons in optically-induced realtime waveguide arrays." Phys. Rev. Lett. 90, 023902 (4 pages) (2003).

7. J. W. Fleischer, M. Segev, N. K. Efremidis, and D. N. Christodoulides. "Observation of two-dimensional discrete solitons in optically-induced nonlinear photonic lattices." Nature 422, 147-150 (2003).

8. D. N. Christodoulides, F. Lederer, and Y. Silberberg. "Discretizing light behaviour in linear and nonlinear waveguide lattices." Nature 424, 817-823 (2003).

9. F. Lederer, G. I. Stegeman, D. N. Christodoulides, G. Assanto, M. Segev, and Y. Silberberg. "Discrete solitons in optics." Phys. Rep. 463, 1-126 (2008).

10. D. N. Christodouldes and E. D. Eugenieva. "Blocking and routing discrete solitons in two-dimensional networks of nonlinear waveguide arrays." Phys. Rev. Lett. 87, 233901 (4 pages) (2001).

11. D. N. Christodouldes and E. D. Eugenieva. "Minimizing bending losses in two-dimensional discrete soliton networks." Opt. Lett. 23 (2001).

12. E. D. Eugenieva, N. Efremidis, and D. N. Christodoulides. "Design of switching junctions for two-dimensional discrete soliton network." Opt. Lett. 26, 1978-1980 (2001). 
13. T. Pertsch, U. Peschel, and F. Lederer. "All-optical switching in quadratically nonlinear waveguide arrays." Opt. Lett. 28, 102-104 (2003).

14. T. Pertsch, U. Peschel, J. Kobelke, K. Schuster, H. Bartelt, S. Nolte, A. Tünnermann, and F. Lederer. "Nonlinearity and disorder in fiber arrays." Phys. Rev. Lett. 93, 053901 (4 pages) (2004).

15. T. Pertsch, U. Peschel, F. Lederer, J. Burghoff, M. Will, S. Nolte, and A. Tünnermann. "Discrete diffraction in two-dimensional arrays of coupled waveguides in silica." Opt. Lett. 29, 468-470 (2004).

16. S. Singh, M. Carvalho, and D. Christodoulides. "Vector interactions of steadystate planar solitons in biased photorefractive media." Opt. Lett. 20, 21772179 (1995).

17. F. Dalfovo, S. Giorgini, L. P. Pitaevskii, and S. Stringari. "Theory of boseeinstein condensation in trapped gases." Rev. Mod. Phys. 71, 463-512 (1999).

18. O. Peleg, G. Bartal, B. Freedman, O. Manela, M. Segev, and D. N. Christodoulides. "Conical diffraction and gap solitons in honeycomb photonic lattices." Phys. Rev. Lett. 98, 103901 (4 pages) (2007).

19. C. R. Rosberg, D. N. Neshev, A. A. Sukhorukov, W. Krolikowski, and Y. S. Kivshar. "Observation of nonlinear self-trapping in triangular photonic lattices." Opt. Lett. 32, 397-399 (2007).

20. Y. V. Kartashov, V. A. Vysloukh, and L. Torner. "Rotary solitons in bessel optical lattices." Phys. Rev. Lett. 93, 093904 (4 pages) (2004).

21. X. Wang, Z. Chen, and P. Kevrekidis. "Observation of discrete solitons and soliton rotation in optically induced periodic ring lattices." Phys. Rev. Lett. 96, 083904 (4 pages) (2006).

22. B. Freedman, G. Bartal, M. Segev, R. Lifshitz, D. N. Christodoulides, and J. W. Fleischer. "Wave and defect dynamics in nonlinear photonic quasicrystals." Nature 440, 1166-1169 (2006).

23. C. Kittel. Introduction to Solid State Physics (Wiley, New York, 1986).

24. P. Yeh. Optical waves in layered media (John Wiley and Sons, New York, 1988).

25. N. K. Efremidis and D. N. Christodoulides. "Lattice solitons in Bose-Einstein condensates." Phys. Rev. A 67, 063608 (9 pages) (2003).

26. A. S. Davydov. "The theory of contraction of proteins under their excitation." J. Theor. Biol. 38, 559-569 (1973).

27. W. P. Su, J. R. Schrerffer, and A. J. Heeger. "Solitons in polyacetylene." Phys. Rev. Lett. 42, 1698-1701 (1979).

28. A. J. Sievers and S. Takeno. "Intrinsic localized modes in anharmonic crystals." Phys. Rev. Lett. 61, 970-973 (1988).

29. A. Trombettoni and A. Smerzi. "Discrete Solitons and Breathers with Dilute Bose-Einstein Condensates." Phys. Rev. Lett. 86, 2353-2356 (2001).

30. A. Scott. "Davidov's soliton." Phys. Rep. 217, 1-67 (1992).

31. S. Flach and C. Willis. "Discrete breathers." Phys. Rep. 295, 181-264 (1998).

32. R. Lai and A. Sievers. "Nonlinear nanoscale localization of magnetic excitations in atomic lattices." Phys. Rep. 314, 148-236 (1999).

33. P. Kevrekidis, B. Malomed, and Y. B. Gaididei. "Solitons in triangular and honeycomb dynamical lattices with the cubic nonlinearity." Phys. Rev. E. 66, 016609 (10 pages) (2002).

34. H. S. Eisenberg, Y. Silberberg, R. Morandotti, and J. S. Aitchison. "Diffraction management." Phys. Rev. Lett. 85, 1863-1866 (2000). 
35. T. Pertsh, T. Zentgraf, U. Peschel, A. Bräuer, and F. Lederer. "Anomalous refraction and diffraction in discrete optical systems." Phys. Rev. Lett. 88, 093901 (4 pages) (2002).

36. D. Dunlap and V. Kenkre. "Dynamic localization of a charged particle moving under the influence of an electric field." Phys. Rev. B 34, 3625-3633 (1986).

37. F. Bloch. "über die quantenmechanik der elektronen in kristallgittern." Z. Phys. 52, 555-600 (1928).

38. U. Peschel, T. Persch, and F. Lederer. "Optical bloch oscillations in waveguide arrays." Opt. Lett. 23, 1701-1703 (1998).

39. T. Pertsch, P. Dannberg, W. Elflein, A. Bräuer, and F. Lederer. "Optical bloch oscillations in temperature tuned waveguide arrays." Phys. Rev. Lett. 83, 4752-4755 (1999).

40. R. Morandotti, U. Peschel, J. S. Aitchison, H. S. Eisenberg, and Y. Silberberg. "Experimental observation of linear and nonlinear optical bloch oscillations." Phys. Rev. Lett. 83, 4756-4759 (1999).

41. G. Lenz, R. Parker, M. Wanke, and C. de Sterke. "Dynamical localization and ac bloch oscillations in periodic optical waveguide arrays." Opt. Commun. 218, 87-92 (2003).

42. S. Longhi. "Self-imaging and modulational instability in an array of periodically curved waveguides." Opt. Lett. 30, 2137-2139 (2005).

43. S. Longhi, M. Marangoni, M. Lobino, R. Ramponi, P. Laporta, E. Cianci, and V. Foglietti. "Observation of dynamic localization in periodically curvedwaveguide arrays." Phys. Rev. Lett. 96, 243901 (4 pages) (2006).

44. R. Iyer, J. S. Aitchison, J. Wan, M. M. Dignam, and C. M. de Sterke. "Exact dynamic localization in curved algaas optical waveguide arrays." Opt. Express 15, 3112-3223 (2007).

45. G. Floquet. "Sur les équations différentielles linéaires à coefficients périodiques." Ann. École Norm. Sup. 12, 47-88 (1883).

46. R. d. L. Kronig and W. G. Penney. "Quantum mechanics of electrons in crystal lattices." P. Roy. Soc. Lond. A Mat. 814, 499-513 (1931).

47. J. Slater. "A soluble problem in energy bands." Phys. Rev. 87, 807-835 (1952).

48. D. Mandelik, H. S. Eisenberg, Y. Silberberg, R. Morandotti, and J. S. Aitchison. "Band-gap structure of waveguide arrays and excitation of floquet-bloch solitons." Phys. Rev. Lett. 90, 053902 (4 pages) (2003).

49. N. K. Efremidis, J. Hudock, D. N. Christodoulides, J. W. Fleischer, O. Cohen, and M. Segev. "Two-dimensional optical lattice solitons." Phys. Rev. Lett. 91, 213906 (4 pages) (2003).

50. C. Zener. "A theory of the electrical breakdown of solid dielectrics." P. Roy. Soc. Lond. A Mat. 145, 523-529 (1934).

51. H. Trompeter, T. Pertsch, F. Lederer, D. Michaelis, U. Streppel, and A. Bräuer. "Visual observation of zener tunneling." Phys. Rev. Lett. 96, 023901 (4 pages) (2006).

52. H. Trompeter, W. Krolikowski, D. N. Neshev, A. S. Desyatnikov, A. A. Sukhorukov, Y. S. Kivshar, T. Pertsch, U. Peschel, and F. Lederer. "Bloch oscillations and zener tunneling in two-dimensional photonic lattices." Phys. Rev. Lett. 96, 053903 (4 pages) (2006).

53. O. Cohen, B. Freedman, J. W. Fleischer, M. Segev, and D. N. Christodoulides. "Grating-mediated waveguiding." Phys. Rev. Lett. 93, 103902 (4 pages) (2004). 
54. O. Manela, M. Segev, and D. N. Christodoulides. "Nondiffracting beams in periodic media." Opt. Lett. 30, 261-263 (2005).

55. M. Ablowitz and J. Ladik. "Nonlinear differential-difference equations and fourier analysis." J. Math. Phys. 17, 1011-1018 (1976).

56. Y. S. Kivshar and D. K. Campbell. "Peierls-nabaro potential barrier for highly localized nonlinear modes." Phys. Rev. E. 48, 3077-3081 (1993).

57. S. Darmanyan, A. Kobyabov, and F. Lederer. "Stability of strongly localized excitations in discrete media with cubic nonlinearity." JETP 86, 682-686 (1998).

58. P. G. Kevrekidis, K. O. Rasmussen, and A. R. Bishop. "The discrete nonlinear schrödinger equation: A survey of recent results." Int. J. Mod. Phys. B 15, 2833-2900 (2001).

59. Y. S. Kivshar. "Self-localization in arrays of defocusing waveguides." Opt. Lett. 14, 1147-1149 (1993).

60. R. MacKay and S. Aubry. "Proof of the existence of breathers for timereversible or Hamiltonian networks of weakly coupled oscillators." Nonlinearity 6, 1623-1643 (1994).

61. P. J. Y. Louis, E. A. Ostrovskaya, C. M. Savage, and Y. S. Kivshar. "Boseeinstein condensates in optical lattices: Band-gap structure and solitons." Phys. Rev. A 67, 013602 (9 pages) (2003).

62. D. Neshev, E. Ostrovskaya, Y. Kivshar, and W. Krolikowski. "Spatial solitons in optically induced gratings." Opt. Lett. 28, 710-712 (2003).

63. D. Mandelik, R. Morandotti, J. S. Aitchison, and Y. Silberberg. "Gap solitons in waveguide arrays." Phys. Rev. Lett. 92, 093904 (4 pages) (2004).

64. A. A. Sukhorukov, D. Neshev, W. Krolikowski, and Y. S. Kivshar. "Nonlinear bloch-wave interaction and bragg scattering in optically induced lattices." Phys. Rev. Lett. 92, 093901 (4 pages) (2004).

65. D. Neshev, A. A. Sukhorukov, B. Hanna, W. Krolikowski, and Y. S. Kivshar. "Controlled generation and steering of spatial gap solitons." Phys. Rev. Lett. 93, 083905 (4 pages) (2004).

66. Y. S. Kivshar and M. Salerno. "Modulational instabilities in the discrete nonlinear schrödinger equation." Phys. Rev. E 49, 3543-3546 (1994).

67. J. Meier, G. I. Stegeman, D. N. Christodoulides, Y. Silberberg, R. Morandotti, H. Yang, G. Salamo, M. Sorel, and J. S. Aitchison. "Experimental observation of discrete modulational instability." Phys. Rev. Lett. 92, 163902 (4 pages) (2004).

68. M. Stepić, C. Wirth, C. E. Rüter, and D. Kip. "Observation of modulational instability in discrete media with self-defocusing nonlinearity." Opt. Lett. 31, 247-249 (2006).

69. Y. S. Kivshar, W. Królikowski, and O. A. Chubykalo. "Dark solitons in discrete lattices." Phys. Rev. E 50, 5020-5032 (1994).

70. R. Morandotti, H. S. Eisenberg, M. Silberberg, Y. Sorel, and J. S. Aitchison. "Self-focusing and defocusing in waveguide arrays." Phys. Rev. Lett. 86, 3296-3299 (2001).

71. D. N. Christodoulides and R. I. Joseph. "Vector solitons in birefringent nonlinear dispersive media." Opt. Lett. 13, 53-55 (1988).

72. A. Darmanyan, S. andKobyakov, E. Schmidt, and F. Lederer. "Strongly localized vectorial modes in nonlinear waveguide arrays." Phys. Rev. E 57, 3520-3530 (1998). 
73. J. Meier, J. Hudock, D. Christodoulides, G. Stegeman, Y. Silberberg, R. Morandotti, and J. S. Aitchison. "Discrete vector solitons in kerr nonlinear waveguide arrays." Phys. Rev. Lett. 91, 143907 (4 pages) (2003).

74. O. Cohen, T. Schwartz, J. W. Fleischer, M. Segev, and D. N. Christodoulides. "Multiband vector lattice solitons." Phys. Rev. Lett. 91, 113901 (4 pages) (2003).

75. A. A. Sukhorukov and Y. S. Kivshar. "Multigap discrete vector solitons." Phys. Rev. Lett. 91, 113902 (4 pages) (2003).

76. T. Peschel, U. Peschel, and F. Lederer. "Discrete bright solitary waves in quadratically nonlinear media." Phys. Rev. E 57, 1127-1133 (1998).

77. R. Iwanow, R. Schiek, G. I. Stegeman, T. Pertsch, F. ederer, Y. Min, and W. Sohler. "Observation of discrete quadratic solitons." Phys. Rev. Lett. 93, 113902 (4 pages) (2004).

78. A. B. Aceves, G. G. Luther, C. De Angelis, A. M. Rubenchik, and S. K. Turitsyn. "Energy localizations in nonlinear fiber arrays: collapse-effect compressor." Phys. Rev. Lett. 75, 73-76 (1995).

79. D. Cheskis, S. Bar-Ad, R. Morandotti, J. S. Aitchison, H. S. Eisenberg, Y. Silberberg, and D. Ross. "Strong spatiotemporal localization in a silica nonlinearwaveguide array." Phys. Rev. Lett. 91, 223901 (4 pages) (2003).

80. A. S. Desyatnikov, E. A. Ostrovskaya, Y. S. Kivshar, and C. Denz. "Composite band-gap solitons in nonlinear optically induced lattices." Phys. Rev. Lett. 91, 153902 (4 pages) (2003).

81. H. J. Shin. "Solutions for solitons in nonlinear optically induced lattices." Phys. Rev. E 69, 067602 (4 pages) (2004).

82. Y. Kominis. "Analytical solitary wave solutions of the nonlinear kronig-penney model in photonic structures." Phys. Rev. E 73, 066619 (6 pages) (2006).

83. K. G. Makris, S. Suntsov, D. N. Christodoulides, G. I. Stegeman, and A. Hache. "Discrete surface solitons." Opt. Lett. 30, 2466-2468 (2005).

84. Y. V. Kartashov, V. A. Vysloukh, and L. Torner. "Surface gap solitons." Phys. Rev. Lett. 96, 073901 (4 pages) (2006).

85. S. Suntsov, K. G. Makris, D. N. Christodoulides, G. I. Stegeman, A. Haché, R. Morandotti, H. Yang, G. Salamo, and M. Sorel. "Observation of discrete surface solitons." Phys. Rev. Lett. 96, 063901 (4 pages) (2006).

86. C. R. Rosberg, D. N. Neshev, W. Krolikowski, A. Mitchell, R. A. Vicencio, M. I. Molina, and Y. S. Kivshar. "Observation of surface gap solitons in semiinfinite waveguide arrays." Phys. Rev. Lett. 97, 083901 (4 pages) (2006).

87. R. Morandotti, U. Peschel, J. S. Aitchison, H. S. Eisenberg, and Y. Silberberg. "Dynamics of discrete solitons in optical waveguide arrays." Phys. Rev. Lett. 83, 2726-2729 (1999).

88. M. J. Ablowitz, Z. H. Musslimani, and G. Biondini. "Methods for discrete solitons in nonlinear lattices." Phys. Rev. E 65, 026602 (4 pages) (2002).

89. T. R. O. Melvin, A. R. Champneys, P. G. Kevrekidis, and J. Cuevas. "Radiationless traveling waves in saturable nonlinear schrödinger lattices." Phys. Rev. Lett. 97, 124101 (4 pages) (2006).

90. D. N. Christodoulides, N. K. Efremidis, P. Di Trapani, and B. A. Malomed. "Bessel x waves in two- and three-dimensional bidispersive optical systems." Opt. Lett. 29, 1446-1448 (2003).

91. Y. Lahini, E. Frumker, Y. Silberberg, S. Droulias, K. Hizanidis, R. Morandotti, and D. N. Christodoulides. "Discrete $\mathrm{x}$-wave formation in nonlinearwaveguide arrays." Phys. Rev. Lett. 98, 023901 (4 pages) (2007). 
92. F. Fedele, J. Yang, and Z. Chen. "Defect modes in one-dimensional photonic lattices." Opt. Lett. 30, 1506-1508 (2005).

93. N. K. Efremidis and K. Hizanidis. "Bandgap lattices: low index solitons and linear properties." Opt. Express 13, 10571-10588 (2005).

94. N. K. Efremidis and D. N. Christodoulides. "Discrete ginzburg-landau solitons." Phys. Rev. E 67, 026606 (5 pages) (2003).

95. E. A. Ultanir, G. I. Stegeman, and D. N. Christodoulides. "Dissipative photonic lattice solitons." Opt. Lett. 29, 845-847 (2004).

96. K.-i. Maruno, A. Ankiewicz, and N. Akhmediev. "Exact localized and periodic solutions of the discrete complex Ginzburg-Landau equation." Opt. Commun. 221, 199-209 (2003).

97. J. Meier, G. I. Stegeman, Y. Silberberg, R. Morandotti, and J. S. Aitchison. "Nonlinear optical beam interactions inwaveguide arrays." Phys. Rev. Lett. 93, 093903 (4 pages) (2004).

98. M. J. Ablowitz and Musslimani. "Discrete diffraction managed spatial solitons." Phys. Rev. Lett. 87 (2001).

99. G. Assanto, A. Fratalocchi, and M. Peccianti. "Spatial solitons in nematic liquid crystals: from bulk to discrete." Opt. Express 15, 5248-5259 (2007).

100. A. B. Aceves, C. De Angelis, T. Peschel, R. Muschall, F. Lederer, S. Trillo, and S. Wabnitz. "Discrete self-trapping, soliton interactions, and beam steering in nonlinear waveguide arrays." Phys. Rev. E 53, 1172-1189 (1996).

101. H. S. Eisenberg, R. Morandotti, Y. Silberberg, J. M. Arnold, G. Pennelli, and J. S. Aitchison. "Optical discrete solitons in waveguide arrays. I. soliton formation." J. Opt. Soc. Am. B 19, 2938-2944 (2002).

102. U. Peschel, R. Morandotti, J. M. Arnold, J. S. Aitchison, H. S. Eisenberg, Y. Silberberg, T. Pertsch, and F. Lederer. "Optical discrete solitons in waveguide arrays. 2. dynamic properties." J. Opt. Soc. Am. B 19, 2637-2644 (2002).

103. V. K. Mezentsev, S. L. Musher, I. V. Ryzhenkova, and S. K. Turitsyn. "Twodimensional solitons in discrete systems." JETP Lett. 60, 829-835 (1994).

104. S. Flach, K. Kladko, and R. S. MacKay. "Energy tresholds for discrete breathers in one-, two-, and three-dimensional lattices." Phys. Rev. Lett. 78, 1207-1210 (1997).

105. M. I. Weinstein. "Excitation thresholds for nonlinear localized modes on lattices." Nonlinearity 12, 673-691 (1999).

106. N. G. Vakhitov and A. A. Kolokolov. "Stationary solutions of the wave equation in a medium with nonlinearity saturation." Radiophys. Quantum Electron. 16, 783-789 (1973).

107. E. Laedke, K. Spatschek, and S. Turitsyn. "Stability of discrete solitons and quasicollapse to intrinsically localized modes." Phys. Rev. Lett. 73, 1055-1059 (1994).

108. Y. Sivan, G. Fibich, N. K. Efremidis, and S. Bar-Ad. "Analytic theory of narrow lattice solitons." Nonlinearity 21, 509-536 (2008).

109. J. Yang and Z. H. Musslimani. "Fundamental and vortex solitons in a twodimensional optical lattice." Opt. Lett. 21, 2094-2096 (2003).

110. H. Martin, E. D. Eugenieva, Z. Chen, and D. N. Christodoulides. "Discrete solitons and soliton-induced dislocations in partially coherent photonic lattices." Phys. Rev. Lett. 92, 123902 (4 pages) (2004).

111. R. Fischer, D. Träger, D. N. Neshev, A. A. Sukhorukov, W. Krolikowski, C. Denz, and Y. S. Kivshar. "Reduced-symmetry two-dimensional solitons in photonic lattices." Phys. Rev. Lett. 96, 023905 (4 pages) (2006). 
112. Y. V. Kartashov, A. A. Egorov, V. A. Vysloukh, and L. Torner. "Surface vortex solitons." Opt. Express 14, 4049-4057 (2006).

113. K. G. Makris, J. Hudock, D. N. Christodoulides, G. I. Stegeman, O. Manela, and M. Segev. "Surface lattice solitons." Opt. Lett. 31, 2774-2776 (2006).

114. X. Wang, A. Bezryadina, Z. Chen, K. G. Makris, D. N. Christodoulides, and G. I. Stegeman. "Observation of two-dimensional surface solitons." Phys. Rev. Lett. 98, 123903 (4 pages) (2007).

115. A. Szameit, Y. Kartashov, F. Dreisow, T. Pertsch, S. Nolte, A. Tünnermann, and L. Torner. "Observation of two-dimensional surface solitons in asymmetric waveguide arrays." Phys. Rev. Lett. 98, 173903 (4 pages) (2007).

116. N. K. Efremidis, D. N. Christodoulides, and K. Hizanidis. "Two-dimensional discrete ginzburg-landau solitons." Phys. Rev. A 76, 043839 (6 pages) (2007).

117. J. Yang, I. Makasyuk, A. Bezryadina, and Z. Chen. "Dipole solitons in optically induced two-dimensional photonic lattices." Opt. Lett. 29, 1662-1664 (2004).

118. Z. Chen, A. Bezryadina, I. Makasyuk, and J. Yang. "Observation of twodimensional lattice vector solitons." Opt. Lett. 29, 1656-1658 (2004).

119. T. Schwartz, G. Bartal, S. Fishman, and M. Segev. "Transport and anderson localization in disordered two-dimensional photonic lattices." Nature 446 , 52-55 (2007).

120. B. A. Malomed and P. G. Kevrekidis. "Discrete vortex solitons." Prys. Rev. E 64, 026601 (6 pages) (2001).

121. B. B. Baizakov, B. A. Malomed, and M. Salerno. "Multidimensional solitons in periodic potentials." Europhys. Lett. 63, 642-648 (2003).

122. J. W. Fleischer, G. Bartal, O. Cohen, O. Manela, M. Segev, J. Hudock, and D. N. Christodoulides. "Observation of vortex-ring "discrete" solitons in $2 \mathrm{~d}$ photonic lattices." Phys. Rev. Lett. 92, 123904 (4 pages) (2004).

123. D. N. Neshev, T. J. Alexander, E. A. Ostrovskaya, Y. S. Kivshar, H. Martin, I. Makasyuk, and Z. Chen. "Observation of discrete vortex solitons in optically induced photonic lattices." Phys. Rev. Lett. 92, 123903 (4 pages) (2004).

124. O. Manela, O. Cohen, G. Bartal, J. W. Fleischer, and M. Segev. "Twodimensional higher-band vortex lattice solitons." Opt. Lett. 29, 2049-2051 (2004).

125. G. Bartal, O. Manela, O. Cohen, J. W. Fleischer, and M. Segev. "Observation of second-band vortex solitons in 2 d photonic lattices." Phys. Rev. Lett. 95, 053904 (4 pages) (2005).

126. T. J. Alexander, A. A. Sukhorukov, and Y. S. Kivshar. "Asymmetric vortex solitons in nonlinear periodic lattices." Phys. Rev. Lett. 93, 063901 (4 pages) (2004).

127. T. J. Alexander, A. S. Desyatnikov, and Y. S. Kivshar. "Multivortex solitons in triangular photonic lattices." Opt. Lett. 32, 1293-1295 (2007).

128. H. Buljan, O. Cohen, J. W. Fleischer, T. Schwartz, M. Segev, Z. H. Musslimani, N. K. Efremidis, and D. N. Christodoulides. "Random-phase solitons in nonlinear periodic lattices." Phys. Rev. Lett 92, 223901 (4 pages) (2004).

129. O. Cohen, G. Bartal, H. Buljan, T. Carmon, J. W. Fleischer, M. Segev, and D. N. Christodoulides. "Observation of random-phase lattice solitons." Nature 433, 500-503 (2005).

130. G. Bartal, O. Cohen, H. Buljan, J. W. Fleischer, O. Manela, and M. Segev. "Brillouin zone spectroscopy of nonlinear photonic lattices." Phys. Rev. Lett. 94, 163902 (4 pages) (2005). 
131. H. Buljan, G. Bartal, O. Cohen, T. Schwartz, O. Manela, T. Carmon, M. Segev, J. W. Fleischer, and D. N. Christodoulides. "Partially coherent waves in nonlinear periodic lattices." Stud. Appl. Math. 115, 173-208 (2005). 